\title{
Spinoptics: Spin degeneracy removal in nanostructures
}

\section{Vladimir Kleiner, Nir Shitrit, Erez Hasman}

Vladimir Kleiner, Nir Shitrit, Erez Hasman, "Spinoptics: Spin degeneracy removal in nanostructures," Proc. SPIE 8269, Photonic and Phononic Properties of Engineered Nanostructures II, 826902 (21 February 2012); doi: $10.1117 / 12.927373$

SPIE. Event: SPIE OPTO, 2012, San Francisco, California, United States 


\title{
Spinoptics: Spin degeneracy removal in nanostructures
}

\author{
Vladimir Kleiner, Nir Shitrit, and Erez Hasman* \\ Micro and Nanooptics Laboratory, Faculty of Mechanical Engineering, and Russell Berrie \\ Nanotechnology Institute, Technion-Israel Institute of Technology, Haifa 32000, Israel \\ *mehasman@technion.ac.il \\ web site: www.technion.ac.il/optics
}

\begin{abstract}
Spin-symmetry breaking in nanoscale structures caused by spin-orbit interaction, leading to a new branch in optics spinoptics is presented. The spin-based effects offer an unprecedented ability to control light and its polarization state in nanometer-scale optical devices, thereby facilitating a variety of applications related to nano-photonics. The direct observation of optical spin-Hall effect that appears when a wave carrying spin angular momentum (AM) interacts with plasmonic nanostructures is introduced. A plasmonic nanostructure exhibits a crucial role of an AM selection rule in a light-surface plasmon scattering process. A spin-dependent dispersion splitting was obtained in a structure consisting of a coupled thermal antenna array. The observed effects inspire one to investigate other spin-based plasmonic effects and to propose a new generation of optical elements for nano-photonic applications.
\end{abstract}

Keywords: nanoaperture, plasmon-polariton, spin-orbit interaction, geometric phase, optical spin-Hall effect

\section{INTRODUCTION}

The interaction of light with metallic subwavelength structures exhibits various anomalous effects such as extraordinary optical transmission and beaming. These effects have been elegantly explained by a mechanism involving the coupling of light to collective surface-confined electronic oscillations known as surface plasmon-polaritons (SPPs). Extensive research has been carried out in the field of electromagnetic surface waves due to its technological potential and fundamental implications. Apparently, the handedness of the light's polarization (optical spin up/down) may provide an additional degree of freedom in nanoscale photonics. The dynamics of spinning light was investigated, and the effect of spin on the trajectories of polarized light beams (spin-orbit coupling) was experimentally observed, with results that agree with the predictions of Berry's phase theory ${ }^{1}$. We examine the spin-orbit coupling effects that appear when a wave carrying intrinsic angular momentum (spin) interacts with a nanoscale structures. The Berry's phase is shown to be a manifestation of the Coriolis effect in noninertial reference frame attached to the wave. The theory is supported by experiment demonstrating the spin-orbit coupling of electromagnetic waves via a surface plasmon anisotropic inhomogeneous nanostructure. The measurements verify the unified geometric phase, demonstrated by the observed polarization-dependent deflection (spin-Hall effect) of the waves ${ }^{2,3}$.

Photonic and Phononic Properties of Engineered Nanostructures II,

edited by Ali Adibi, Shawn-Yu Lin, Axel Scherer, Proc of SPIE Vol. 8269

$826902 \cdot$ (C) 2012 SPIE · CCC code: 0277-786X/12/\$18 - doi: 10.1117/12.927373

Proc. of SPIE Vol. $8269826902-1$ 


\section{OBSERVATION OF THE SPIN-BASED PLASMONIC EFFECT IN NANOSCALE STRUCTURES}

The proposed anisotropic inhomogeneous plasmonic structure was produced on top of a thin metal film evaporated onto a glass plate. The element consisted of a cavity (a spiral Bragg grating with a central defect), surrounded by a coupling grating (Fig. 1(a)). The structure was illuminated by circularly polarized light ( $\mathbf{R}-$ right-handed, $\mathbf{L}-$ left handed). The intensity in the plasmonic cavity was measured by a Near-field Scanning Optical Microscope (NSOM). The measured intensity distribution exhibits a strong dependence on the incident spin (see Fig. 1(a)). An annular ring structure with a dark spot in the center for $\mathbf{R}$ illumination and with a bright spot for $\mathbf{L}$ illumination indicates coupling to different spiral plasmonic modes. The origin of the spin-dependent change in the near-field intensity distributions lies in the geometric phase of the excited plasmonic mode. In the most general case, when a wave carrying an arbitrary spin angular momentum changes its direction of propagation and polarization state, the geometric phase is given by a simple expression stemming from the Coriolis effect ${ }^{2}$. The Coriolis effect is a result of the rotation of the reference frame, represented by the local direction of the grating grooves. Accordingly a spiral geometric phase with spin-dependent helicity arises in a circular grating ${ }^{3}$. In the spiral structure, (see Fig. 1(a)) an additional dynamic phase arises as a result of a space-variant path difference. The overall phase in the spiral cavity is the sum of the geometric and dynamic phases, which is manifested by different spiral modes that are obtained in the cavity for different polarizations.

One of the possible technological implementations of the plasmonic geometric phase could be a spin-dependent plasmonic focusing lens. The proposed structure is presented in Figure 1(b). This structure was illuminated from the bottom with $\mathbf{R}$ and $\mathbf{L}-$ polarized plane waves and the near-field intensity distribution was collected by the NSOM. A spin-

(a)

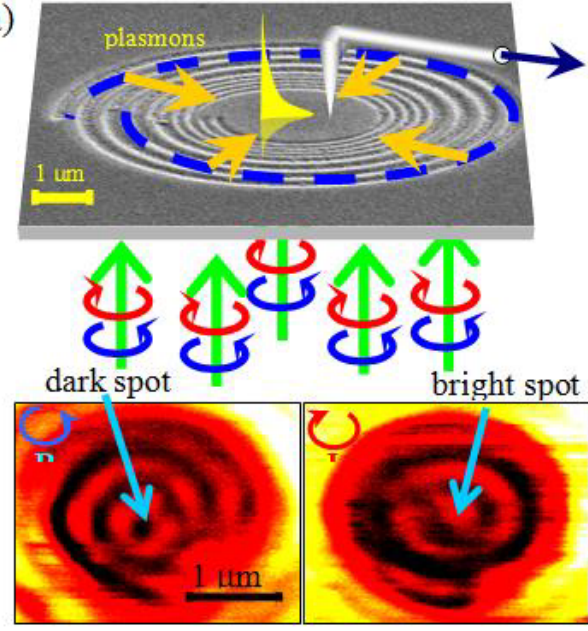

(b)
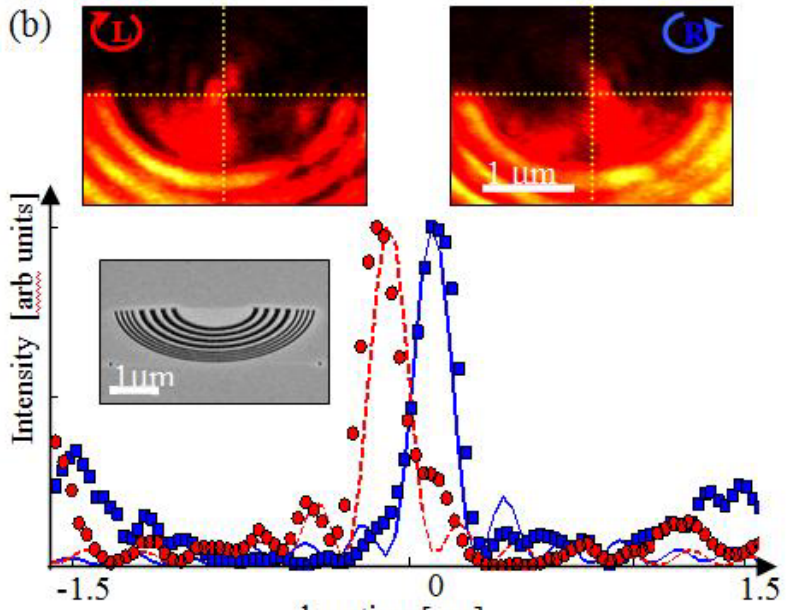

location $[\mu \mathrm{m}]$

Figure 1. (a) The scanning electron microscope image of the spiral nanostructure and the scheme of the optical setup. Intensity distribution in the cavity measured by a NSOM for $\mathbf{R}$ and $\mathbf{L}$ illumination. (b) Spin-dependent plasmonic lens based on a plasmonic spin-Hall effect. The intensity distributions measured by a NSOM for $\mathbf{R}$ and $\mathbf{L}$ illumination and the corresponding transverse cross-sections of the measured intensity distributions in the focal plane of the lens (R blue squares, $\mathbf{L}$ - red circles ) Calculated cross-sections are plotted for each polarization (solid blue line -R; dashed red line - L). The SEM picture of the element is depicted in the inset.

dependent transverse shift of the focus is observed by comparing the spots (Fig. 1(b)). This shift can be regarded as a manifestation of the optical Magnus effect (optical spin-Hall effect) which arises in our system due to a spiral geometric phase. The observed effects inspire one to investigate other spin-based plasmonic effects and to propose a new generation of optical elements for nano-photonic applications. 


\section{OBSERVATION OF OPTICAL SPIN SYMMETRY BREAKING IN NANOAPERTURES}

Observation of a spin symmetry breaking effect in plasmonic nanoscale-structures due to spin-orbit interaction is presented. We demonstrate a nanoplasmonic structure which exhibits a crucial role of an angular momentum (AM) selection rule in a light-surface plasmon scattering process. In our experiment, the intrinsic AM (spin) of the incident radiation is coupled to the extrinsic momentum (orbital AM) of the surface plasmons via spin-orbit interaction. Due to this effect, we achieved a spin-controlled enhanced transmission through a coaxial nanoaperture ${ }^{4}$.

In our experiment the spin-orbit interaction mechanism and the AM selection rules were experimentally verified by investigating the effect in a circularly symmetric - achiral - nanostructure. For this purpose we induced an external
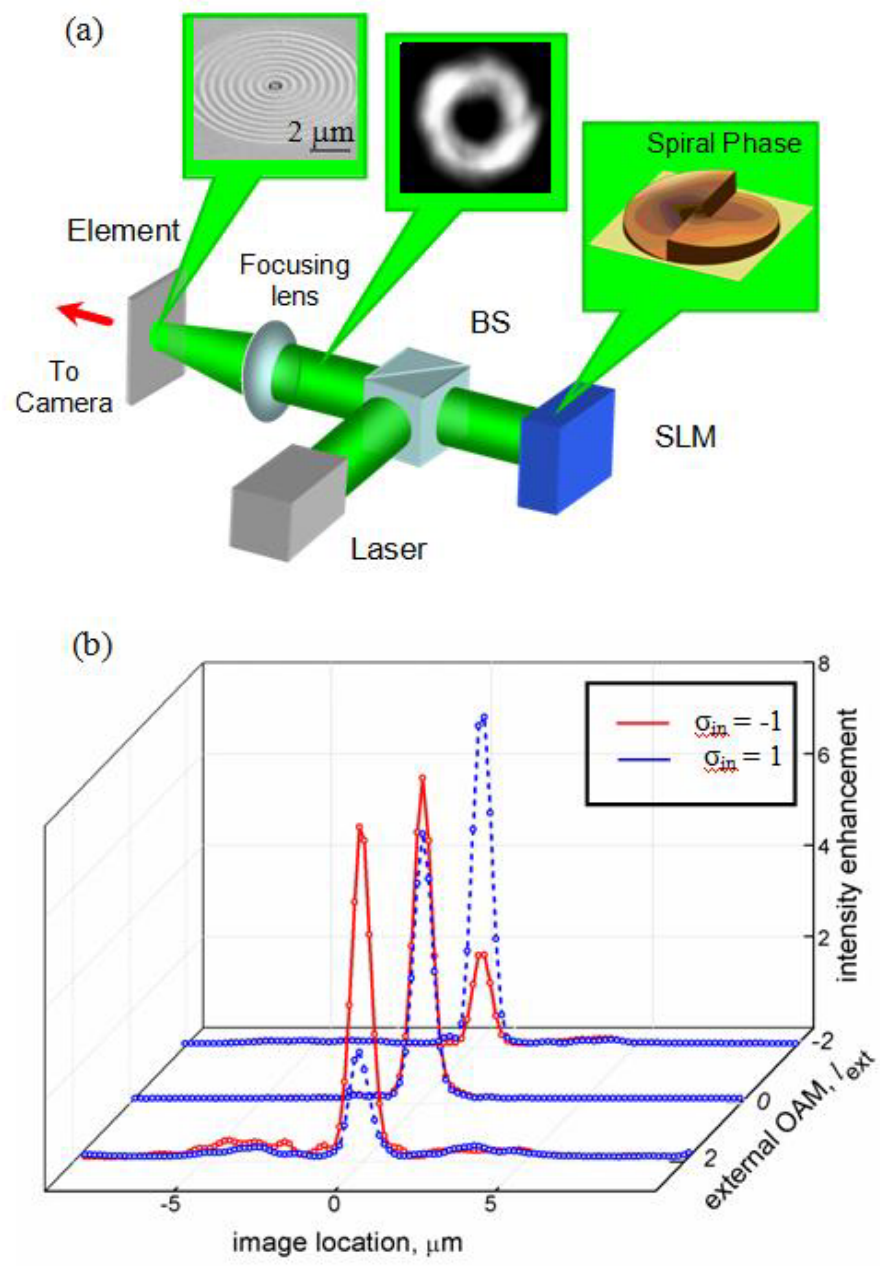

Figure 2. Removal of the spin-degeneracy in circular corrugation by use of externally induced OAM. (a) experimental setup. A laser beam is modulated by a Spatial Light Modulator (SLM) to obtain a spiral phase and then incident through a Beam Splitter (BS) onto a coaxial aperture with circular corrugation. The transmitted light is captured in the image plane by the camera. The spiral phase with $l_{e x t}=2$, the measured intensity distribution across the incident beam, and the SEM picture of the element are presented in the figure. (b) intensity distribution cross-sections captured by the camera for different $l_{\text {ext }}$. 
OAM carried by the incident beam. We fabricated the element, which consisted of an individual coaxial aperture, surrounded by an annular coupling grating (see Fig. 2a). This aperture was illuminated by a green laser light at a wavelength $532 \mathrm{~nm}$ whose phase was modulated by a spatial light modulator (to achieve a spiral phase with topological charges $l_{\text {ext }}=0, \pm 2$. We obtained a spin-dependent enhanced transmission by controlling the behavior of the device with external orbital AM, (see Fig. 2(b)).

\section{PLASMONIC AHARONOV-BOHM EFFECT}

A circular nanoslit was used to excite an out-propagating plasmonic wavefront. We measured the scattered plasmonicwave dislocation strength by its interference with an additional plasmonic reference wave (see Fig. 3). The dislocation strength was shown to be equal to the incident optical spin (polarization helicity) in a manner similar to the AharonovBohm wavefunction dislocation strength being equal to the magnetic flux parameter. Moreover, we experimentally demonstrated that the surface plasmon-polariton wave dislocation is independent on the incident wavelength and the nanoslit diameter, therefore verifying the geometric nature of the phenomenon. This effect is attributed to the optical spin-orbit interaction - coupling of the intrinsic angular momentum - spin and the extrinsic (orbital/linear) momentum of the electromagnetic field 5. Our experiment was analyzed using a rotating reference frame, which leads to a spindependent correction of the momentum term in the wave equation. The experiment and analysis presented in this letter elucidate the significance of the optical spin in the scattering of surface plasmon-polaritons from

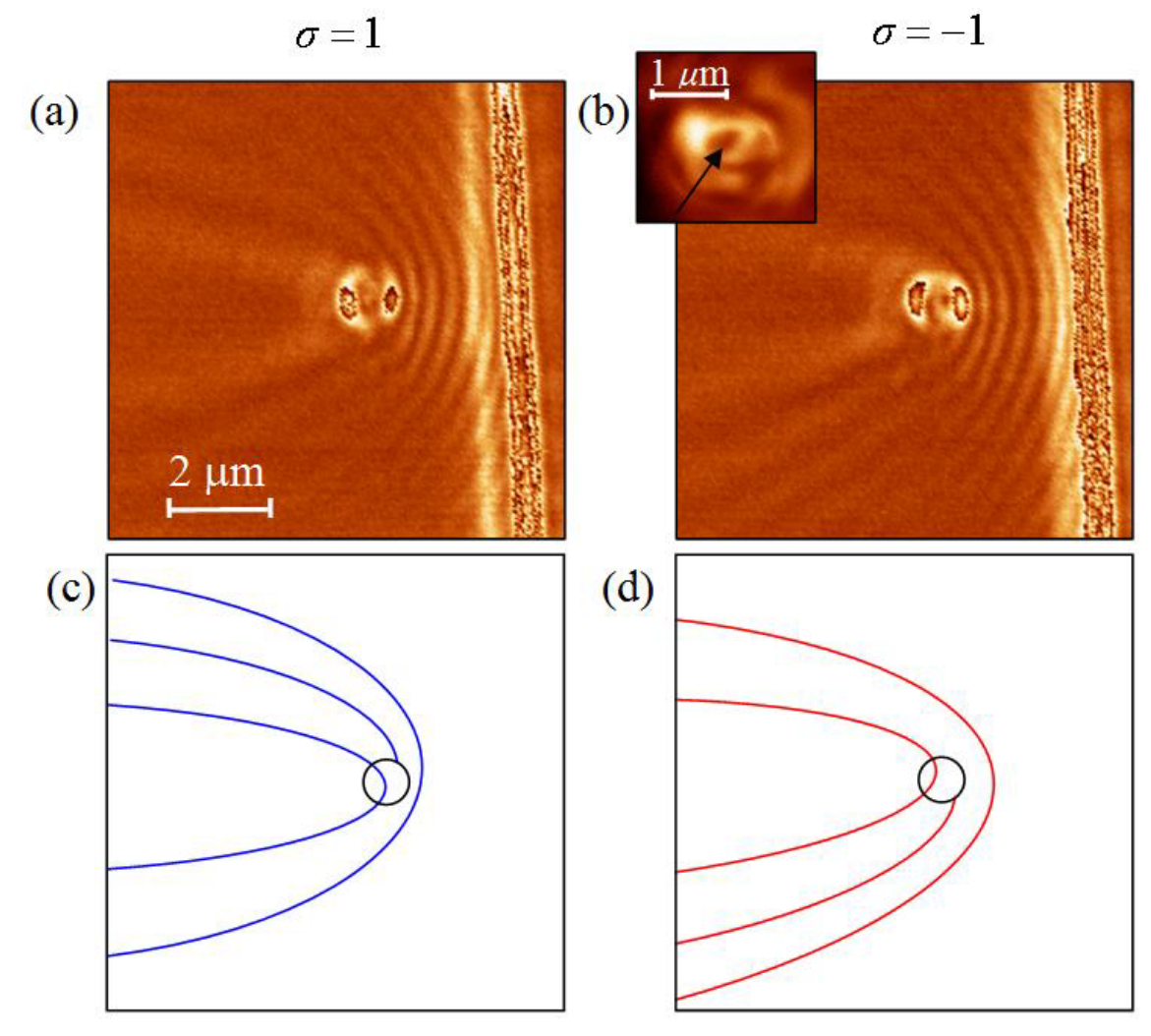

Figure 3. Experimentally measured near-field intensity for (a) $\sigma=1$ polarization (right circular polarization) and (b) $\sigma=-1$ polarization (left circular polarization) at wavelength $800 \mathrm{~nm}$. The measured fringe maxima are presented in (c) for $\sigma=1$ and (d) for $\sigma=-1$ states. The black circle in (c) and (d) represents the location of the circular nanoslit. The inset in (b) with a dark spot in the center (marked with an arrow) is the measured intensity distribution inside the circular slit. 


\section{OPTICAL SPIN-HALL EFFECTS IN PLASMONIC CHAINS}

Spin-Hall effect is a basic phenomenon arising from the spin-orbit coupling of electrons. In particular, the spatial trajectory of the moving electrons is affected by their intrinsic angular momentum. More generally, the dispersion relation of the particles is modified in a spin-dependent manner.6 The spin-Hall effect is regarded as intrinsic when it arises due to Rashba coupling (Berry curvature), while the extrinsic effect occurs due to the spin-orbit-dependent scattering of electrons from impurities. The optical spin-Hall effect (OSHE) - beam displacement and momentum shift

(a)

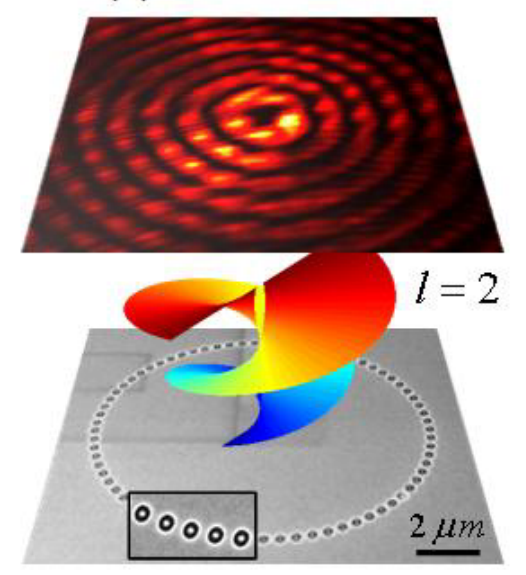

(b)

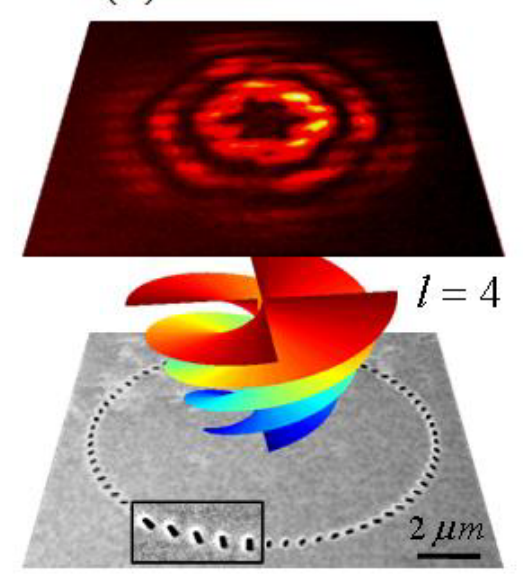

(c)

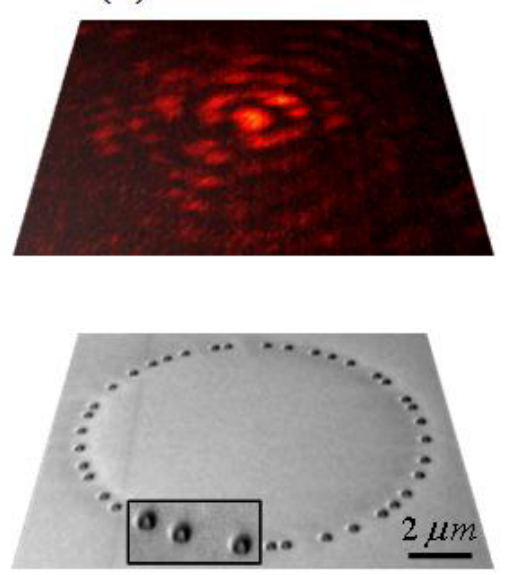

Figure 4. The OSHE-LI and OSHE-LA for circular chains. The measured far-field intensity distribution of the spin-flip component scattered from a circular chain of ordered (a) and disordered (c) coaxial apertures at a wavelength of 780 $\mathrm{nm}$, and rotating nanorods with $m=2$ at a wavelength of $730 \mathrm{~nm}$ (b); bottom, SEM images of the chains with radii of $5 \mu \mathrm{m}$. The spin-Hall momentum deviation is accompanied by a spiral phase-front with the topological charges $l=2$ and $l=4$, for the OSHE-LI (a) and OSHE-LA (b), respectively. Note that no spin-Hall momentum deviation is observed from the disordered chain (c).

due to the optical spin (polarization helicity) - was recently presented. ${ }^{1,7}$ The effect was attributed to the optical spinorbit interaction occurring when the light passes through an anisotropic and inhomogeneous medium. Here, we present and experimentally observe the optical spin-Hall effects in coupled localized plasmonic chains ${ }^{8}$. The locally isotropic optical spin-Hall effect (OSHE-LI) is regarded as the interaction between the optical spin and the path of the plasmonic chain with an isotropic unit cell. In contrast, the locally anisotropic optical spin-Hall effect (OSHE-LA) occurs due to the interaction between the optical spin and the local anisotropy of the unit cell, which is independent on the chain path. This latter mechanism expands the scope of the OSHE and provides an additional degree of freedom in spin-based optics.

The OSHE-LI was studied on a coupled plasmonic chain of annular apertures, whose local orientation varies linearly with the $x$ coordinate. The local orientation of the curved chain induces local anisotropy variation in the scattered field. Polarization analysis shows that the scattering from the curved chain contains a spin-flip component, which is experience a spin-dependent beam deflection. Similar beam deflection was observed in scattering from linear chain of rectangular apertures (nanorodes) with orientation linearly varied along the chain (OSHE-LA). In other experiment the circular chains of coaxial apertures and rotating nanorods demonstrate scattered wave with an orbital angular momentum as result of spin-orbit interaction between light and anisotropic inhomogeneous structure (see Fig. 4). The observed OSHEs are due to the collective interaction of the localized modes within the periodic plasmonic chains. Emergence of spiral phase front with orbital AM was then directly verified by interference experiment and strength of phase singularity was measured. 


\section{SPIN SYMMETRY BREAKING IN THERMAL EMISSION}

When light is emitted or scattered from a revolving medium, it exhibits a dispersion splitting - angular Doppler effect (ADE) - which depends on the circular polarization handedness (the photon's spin) ${ }^{9}$. The dispersion splitting is attributed to a spin-dependent correction of the momentum term in the wave equation due to rotation of the emitting medium. This splitting is the manifestation of the spin-orbit interaction, which is the basis for optical spin-Hall, Magnus, and Coriolis effects. Here, we report on a geometric Doppler effect manifested by a spin-dependent dispersion splitting of thermal radiation emitted from a structure whose local anisotropy is rotated along $x$-axis. The observed effect is attributed to the dynamics of the thermally excited surface wave propagating along the structure ${ }^{10}$.

In our experiment deepenings ("thermal antennas") with the subwavelength size of $1.2 \mu \mathrm{m} \times 4.8 \mu \mathrm{m}$ (Fig. 5, inset(i)) were etched to a depth of $1 \mu \mathrm{m}$ on a SiC substrate forming isolated thermal antenna and coupled thermal antenna array (Fig. 5, inset(iii)) with a period of $\Lambda=11.6 \mu \mathrm{m}$. The upper plot in Fig. 4 is the emission spectrum of the isolated thermal antenna, which shows local resonances at frequencies $890 \mathrm{~cm}^{-1}$ and $945 \mathrm{~cm}^{-1}$. These modes exhibit strong linear

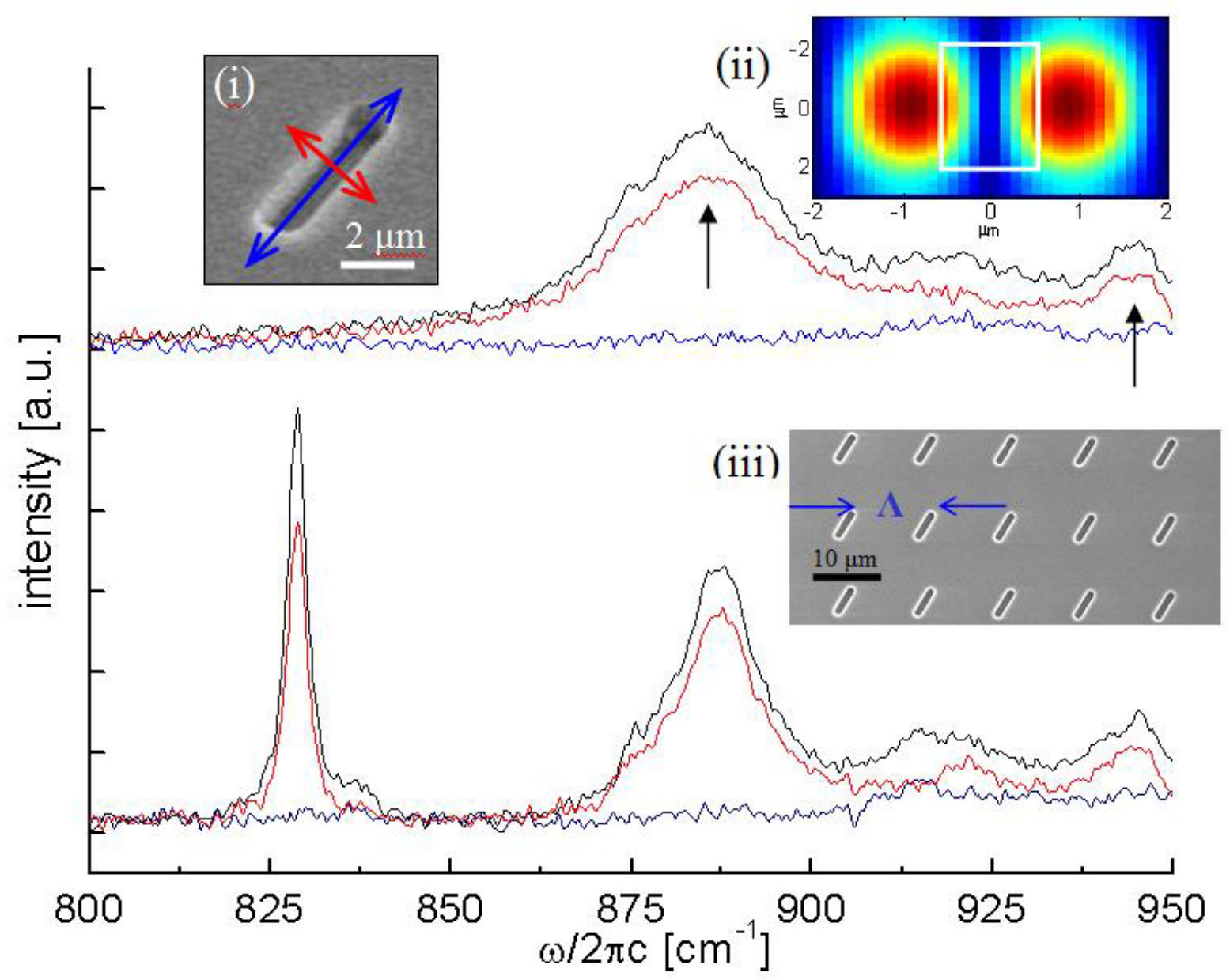

Figure 5. Spectral emission from the isolated thermal antenna (top, SEM image in inset (i)), and for homogeneous coupled oriented antenna array (bottom, SEM image in inset (iii)); red and blue curves correspond to polarization along the short axis and long axis of the antenna respectively, and black for the total intensity. Inset(ii) presents a FDTD simulation of the intensity distribution in vicinity of an isolated antenna at $890 \mathrm{~cm}^{-1}$, white line indicates the location of the antenna. Black arrows point to the local resonances. 
polarization along the direction of the small axis of the antenna. The same polarization of local modes is observed in the emission from the coupled antenna array (Fig. 5, inset (iii)). However, in this case the spectrum at normal incidence, contains an additional narrow peak at $830 \mathrm{~cm}^{-1}$, which is also strongly linear polarized along the same direction (Fig. 5 , bottom). By measuring the emission dispersion from the array of identically oriented antennas, we have found that the narrow spectral peak contains two extended dispersive modes - a fast mode and a slow mode, attributed to coupled, localized surface phonon-polaritons (Fig. 6 (a)). By applying polarization measurements, we verified that the polarization direction of the slow mode follows the antennas' orientation.

Different and very intriguing polarization properties were observed in an array of antennas whose orientation was gradually rotated along the $x$-axis (Fig.6(b), inset). In the measured dispersion of thermal radiation ${ }^{10}$, the slow mode exhibits a clear splitting in the momentum of the emitted waves. The above phenomenon can be elucidated when considering surface waves propagation. As surface phonon-polaritons travel along the superstructure, they radiate a linearly polarized field that rotates at a spatial rate $\Omega=d \phi / d x$ ( $\phi$-local antenna's orientation). This rotation induces coupling between the intrinsic and the extrinsic momentum - spin-orbit interaction - which leads to a spin-dependent perturbation of the momentum $\Delta k=\sigma \Omega$, where $\sigma= \pm 1$ is the spin state. By solving the perturbated Helmholtz equation we derive the dispersion shift $\omega=\omega\left(k_{x}+\sigma \Omega\right)$ in the momentum dimension. Therefore, the original dispersion of the homogeneous structure is split into two emitted modes with opposite spin states. We found the spin-projected dispersion of the thermal emission (Fig. 6(b)) by measuring the Stokes' polarization parameter $S_{3}$, which represents the circular polarization portion. This measurement reveals that the slow mode is split in the momentum by $2 \Omega$ between the two opposite spin-states.

(a)

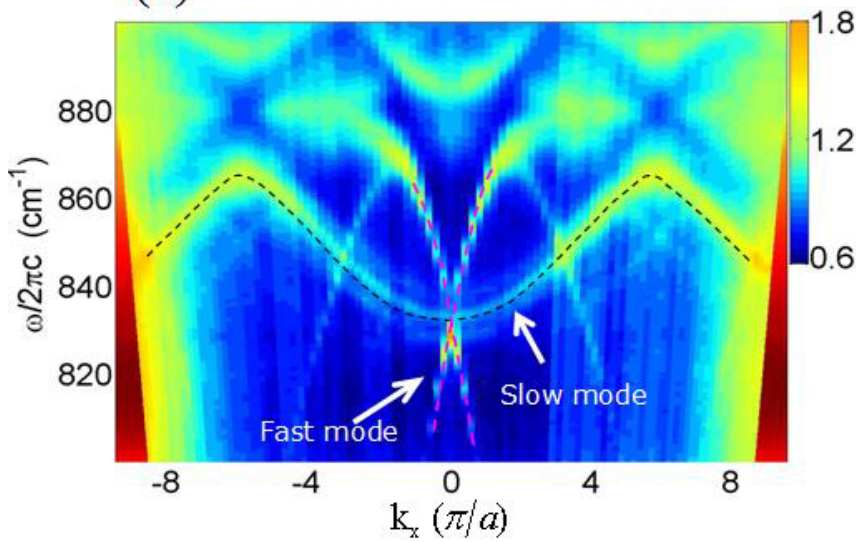

(b)
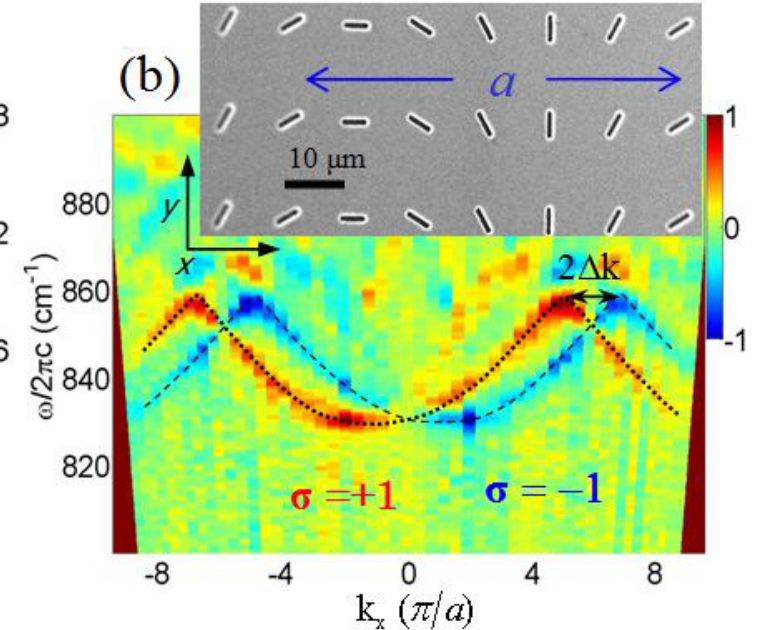

Figure 6. (a) The measured emission dispersion of the coupled thermal antenna array (Fig. 5 inset (iii)). Dashed lines highlight the dispersion of the slow and the fast modes. (b) Spin-projected dispersion of the rotating antenna array, obtained by $S_{3}$ measurement. Dashed/dotted lines highlight the dispersion of the spin-split slow mode (red/blue color corresponds to a positive/negative spin projection, respectively). The observed dispersion shift is $\Delta k=\sigma \Omega$, signs of $\sigma= \pm 1$ correspond to two opposite circular polarizations; in the inset - SEM of the antenna array rotating along the xaxis with a period $a=6 \Lambda ;(\Omega=\pi / a)$.

\section{CONCLUSION}

The spin of photons can provide an additional degree of freedom in nanoscale photonics leading to a new branch in optics - Spinoptics ${ }^{11,12}$. 


\section{REFERENCES}

[1] Bliokh, K. Y., Niv, A., Kleiner, V. and Hasman, E., "Geometrodynamics of spinning light", Nature Photonics 2, 748-753 (2008).

[2] Bliokh K. Y., Gorodetski Y., Kleiner V., and Hasman E., "Coriolis effect in optics: unified geometric phase and spin-Hall effect", Phys. Rev. Lett. 101(3), 030404-1 - 030404-4 (2008)

[3] Gorodetski, Y., Niv, A., Kleiner, V. and Hasman, E., "Observation of the spin-based plasmonic effect in nanoscale structures", Phys. Rev. Lett. 101(4), 043903-1 - 043903-4 (2008).

[4] Gorodetski Y., Shitrit N., Bretner I., Kleiner V., and Hasman E., "Observation of Optical Spin Symmetry Breaking in Nanoapertures", Nano Letters, 9 (8), 3016-3019 (2009).

[5] Gorodetski Y., Nechayev S., Kleiner V., and Hasman E., "Plasmonic Aharonov-Bohm effect: Optical spin as the magnetic flux parameter", Phys. Rev. B 82, 125433-1 - 125433-4(2010)

[6] Bychkov Y. A., Rashba E. I., "Oscillatory effects and the magnetic susceptibility of carriers in inversion layers", J. Phys. C, 17(33), 6039-6045 (1984).

[7] Leyder, C., Romanelli, M., Karr, J. Ph., Giacobino, E., Liew, T. C. H., Glazov, M. M., Kavokin, A. V., Malpuech, G., Bramati, A., "Observation of the optical spin Hall effect", Nature Phys. 3, 628-631, (2007).

[8] Shitrit, N., Bretner I., Gorodetski Y., Kleiner, V. and Hasman E., "Optical Spin Hall Effects in Plasmonic Chains", Nano Letters 11, 2068 (2011).

[9] Garetz, B. A., "Angular Doppler effect", J. Opt. Soc. Am. 71, 609-611 (1981).

[10] Dahan N., Gorodetski Y., Frischwasser K., Kleiner V., and Hasman E., "Geometric Doppler Effect: Spin-Split Dispersion of Thermal Radiation", Phys. Rev. Lett. 105(13), 136402-1 - 136402-4 (2010).

[11] Frischwasser, K., Yulevich, I., Kleiner, V. and Hasman, E., "Rashba-like spin degeneracy breaking in coupled thermal antenna lattices", Opt. Express 19, 23475-23481 (2011).

[12] See additional references at www.technion.ac.il/optics 